neurological examination showed no abnormalities. Spinal MRI showed paravertebral abscess in $56.5 \%$ of cases. Seventeen patients $(73,9 \%)$ had epiduritis and 9 patients $(39,9 \%)$ had spinal cord compression on the MRI. An abnormal signal of the spinal cord was observed in 2 cases $(8,7 \%)$. All patients received a combination of Rifampicin and Doxycycline. The mean duration of the antimicrobial treatment was 8 months. There was no statistically significant association between the occurrence of abscesses, epiduritis, spinal cord compression, abnormal signal of the spinal cord on the MRI and the duration of treatment $(p=0.935$, $p=0.929, p=0.379, p=0.889$ respectively).

Conclusions: MRI of the spine frequently revealed signs of severity in brucellar spondylodiscitis patients, although without clinical expression. Despite their severity these signs did not result in a longer period of antibiotic therapy

Disclosure of Interest: None declared

DOI: 10.1136/annrheumdis-2018-eular.5892

\section{AB1056 DIAGNOSIS VALUE OF PERCUTANEOUS SPINAL NEEDLE BIOPSY IN BRUCELLAR SPONDYLODISCITIS}

O. Saidane, I. Oueslati, I. Mahmoud, A. Ben Tekaya, R. Tekaya, L. Abdelmoula. Rheumatology department, Charles Nicolle hospital, Tunis, Tunisia

Background: spondylodiscitis is a serious condition with a large variety of infectious etiology. Vertebral biopsy is needed to perform the microbiologic diagnosis when no causative organism is identified. CT-guided percutaneous spinal biopsy (CTSB) may reduce the risk of contamination and complications.

Objectives: The aim of this study is to determine the contribution of CTSB to the diagnosis of Brucellar spondylodiscitis.

Methods: we conducted a retrospective study on 27 patients admitted for Brucellar spondylodiscitis over a 17 years period [2000 to 2016]. The etiological diagnosis was based on Brucella agglutination test which was positive for all patients. Twelve patients had a CTSB with cytobacteriological and histological tests.

Results: twenty seven patients (17 men and 10 women) with a mean age of 54 years were included. Twenty six patients $(96,3 \%)$ reported a spinal pain. This pain involved the lumbar spine (59.3\%), the dorsal spine (18.5\%) and less frequently the cervical spine $(11,1 \%)$. Eight patients reported lombosciatica $(29.6 \%)$. An etiological doubt subsisted in 12 cases and a CTSB was performed. Culture results were negative for 11 biopsy samples and one culture was positive to Staphylococcus in a case of a co-infection. The results of the histological examination showed chronic non-specific inflammation in $92.3 \%$ of cases. An infectious etiology was suspected histologically in $53.8 \%$ of cases and particularly of a pyogenic germ (23.1\%). The biopsy was no contributive in one case

Conclusions: Our results suggest that CT-guided spinal biopsy is not useful to diagnose Brucellar spondylodiscitis. However, the absence of tuberculoid granuloma and caseous necrosis helped ruling out the tubercular origin

Disclosure of Interest: None declared

DOI: 10.1136/annrheumdis-2018-eular.5933

\section{AB1057 BRUCELLAR SPONDYLODISCITIS: THE IMAGING FINDINGS}

O. Saidane, I. Oueslati, R. Tekaya, A. Ben Tekaya, I. Mahmoud, L. Abdelmoula Rheumatology department, Charles Nicolle hospital, Tunis, Tunisia

Background: Brucellar spondylodiscitis is an important complication of brucellosis that can cause neurologic involvement and spinal deformities if not appropriately treated. Imaging examination is a major key in diagnosis

Objectives: The aim of this study was to report the imaging findings in brucellar spondylodiscitis

Methods: We performed a retrospective study including 27 patients with Brucellar spondylodiscitis over a period of 17 years from 2000 to 2016. Diagnosis was made on clinical presentation, laboratory findings, radiographic evidence and Brucellar seroagglutination tests. All patients underwent X-rays of the involved spine levels. Spinal computed tomography (CT)was carried out in 13 cases and spinal $\mathrm{MRI}$ in 24 cases

Results: seventeen men and 10 women aged from 33 to 75 years were included. Plain radiographs showed a disc space narrowing for 26 patients $(96,3 \%)$ and an irregularity of the vertebral end plate in 16 cases $(59,3 \%)$. A paravertebral abscess formation was detected for 3 patients. No obvious abnormalities were found in one case. CT scans of most patients revealed signs of spondylodiscitis $(92,3 \%)$. This examination showed an erosion of the vertebral end plates $(69.2 \%)$, intervetebral disc space narrowing $(61,5 \%)$ and bone destruction $(38.5 \%)$. The severity signs detected on the CTs were the soft tissu thickening (46.2\%), abscesses formations $(7.4 \%)$, epiduritis $(30.8 \%)$ and one patient had a spinal cord compression. The 24 MRIs realised exhibited a signal abnormality of the vertebral body $(95.7 \%)$ and the intervertebral disc $(47.8 \%)$ in addition to disc space narrowing $(73.9 \%)$ with erosions of the vertebral end plates $(56.0 \%)$. The contrast enhanced T1- weighted images showed marked enhancement of affected vertebrae and disc (78.3\%). Thirteen patients had abscesses formations $(56,5 \%), 17$ had epiduritis $(73,9 \%)$ and 9 patients $(31,9 \%)$ presented a spinal cord compression on MRI. Bru cellar spondylodiscitis involved one spinal level in 23 cases whereas multilevel involvement was found in the 4 others

Conclusions: plain radiographs and spinal CT scans luck sensitivity in diagnosing brucellar spondylodiscitis and spinal MRI remains the referential imaging modality to recognise early bone infection, allowing complete lesion topography and identifying the complications.

Disclosure of Interest: None declared

DOI: 10.1136/annrheumdis-2018-eular.5913

\section{AB1058 $\quad$ IS HEPATITIS B SURFACE ANTIGEN (HB S AG) ENOUGH ALONE AS A SCREENING TEST FOR HBV INFECTION IN RHEUMATIC DISEASE PATIENTS BEFORE STARTING IMMUNOSUPPRESSIVE THERAPIES?}

R.A. Abdel Noor ${ }^{1}$, M. Watany ${ }^{2}$, S. Abd-Elsalam³ ${ }^{3}$, W. Elkhalawany ${ }^{3}$, S. Soliman ${ }^{4}$, R. Badawi ${ }^{3} .{ }^{1}$ Internal MedicineandRheumatology; ${ }^{2}$ clinical pathology; ${ }^{3}$ Tropical Medicine, Faculty of Medicine, Tanta University, Tanta; ${ }^{4}$ public healthandcommunity medicine, Faculty of Medicine, Menofia university, Menofia, Egypt

Background: Prevalence of hepatitis B virus in patients with rheumatic diseases has been reported differently among studies. The loss of immune control in these patients may result in the reactivation of HBV replication within hepatocytes. Con sidering the lifelong use of multiple anti-rheumatic drugs, screening for HBV is recommended before starting immunosuppressive or immunomodulatory therapy.

Objectives: The aim of this study was to select the best and simplest test for screening of HBV in rheumatic patients in Egypt.

Methods: This cross sectional study was carried out on 102 patients with different rheumatic diseases. Screening to all patients by hepatitis B surface antigen (HBsAg), hepatitis $\mathrm{C}$ virus (HCV) and human immune deficiency virus antibodies (HIV) were done. HBV core antibodies and real time PCR to detect HBV DNA were done.

Results: The mean age of the patients was $37.18 \% \pm 12.37 .3 .9 \%$ of them were males and $96.1 \%$ were females. We found that HBsAg was positive in two patients $(2 \%)$ and negative in 100 patients $(98 \%)$. HBcAb was positive in 24 patients $(23.5 \%)$ and negative in 78 patients. however PCR for hepatitis B DNA was positive in 2 patients (2.0\%) only who were positive for both $\mathrm{HBs} \mathrm{Ag}$ and $\mathrm{HBC}$ Ab. HBsAg had 100\% Sensitivity, 100\% Specificity, 100\% PPV, 100\% NPV and $99.0 \%$ accuracy. While antiHBc had $100 \%$ Sensitivity, $78 \%$ Specificity, $8 \%$ PPV $100 \%$ NPV and $78 \%$ accuracy in screening of HBV.

Abstract AB1058 - Table 1. Serological diagnosis of HBV in rheumatic disease patients

\begin{tabular}{lccc}
\hline & Negative & Positive & Total $(n=102)$ \\
& No\% & No\% & No\% \\
\hline Anti-HBc: & 7876.5 & 2423.5 & 102100.0 \\
HBs Ag & 10098.0 & 22.0 & 102100.0 \\
PCR & 10098.0 & 22.0 & 102100.0 \\
\hline
\end{tabular}

Abstract AB1058 - Table 2. The validity of HBsAg and HBcAb in relation to HBv DNA by PCR

\begin{tabular}{|c|c|c|c|c|}
\hline \multicolumn{5}{|c|}{ validity of $\mathrm{HBs}-\mathrm{Ag}$ in relation to $\mathrm{PCR}$ in diagnosis of $\mathrm{HBV}$} \\
\hline Sensitivity & Specificity & PPV & NPV & Accuracy \\
\hline $100.0 \%$ & $100.0 \%$ & $100.0 \%$ & $100.0 \%$ & $99.0 \%$ \\
\hline \multicolumn{5}{|c|}{ validity of AnitHBc $A b$ in relation to PCR in diagnosis of HBV } \\
\hline Sensitivity & Specificity & PPV & NPV & Accuracy \\
\hline $100.0 \%$ & $78.0 \%$ & $8.0 \%$ & $100.0 \%$ & $78.0 \%$ \\
\hline
\end{tabular}

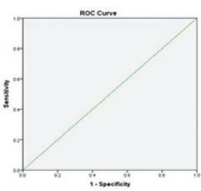

HBs A

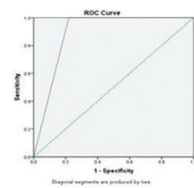

$\mathrm{HBC} A \mathrm{~b}$
Abstract AB1058 - Figure 1. Receiver operating characteristic (ROC) plot for "HBs Ag in relation to PCR in diagnosing HBV" on the left side \& "HB c Ab in relation to PCR" on the right side. 